\title{
Substrate-induced chiral states in graphene
}

\author{
M. Zarenia, O. Leenaerts, B. Partoens, and F. M. Peeters \\ Department of Physics, University of Antwerp, Groenenborgerlaan 171, B-2020 Antwerpen, Belgium
}

(Received 9 February 2012; revised manuscript received 13 June 2012; published 27 August 2012)

\begin{abstract}
Unidirectional chiral states are predicted in single layer graphene which originate from the breaking of the sublattice symmetry due to an asymmetric mass potential. The latter can be created experimentally using boron-nitride $(\mathrm{BN})$ substrates with a line defect $(\mathrm{B}-\mathrm{B}$ or $\mathrm{N}-\mathrm{N})$ that changes the induced mass potential in graphene. Solving the Dirac-Weyl equation, the obtained energy spectrum is compared with the one calculated using $a b$ initio density functional calculations. We found that these one-dimensional chiral states are very robust and they can even exist in the presence of a small gap between the mass regions. In the latter case additional bound states are found that are topologically different from those chiral states.
\end{abstract}

DOI: 10.1103/PhysRevB.86.085451

PACS number(s): 71.10.Pm, 73.21.-b, 81.05.ue

\section{INTRODUCTION}

The discovery of graphene, i.e., a single layer of carbon atoms with honeycomb structure, introduced an interesting novel material to the condensed-matter community. ${ }^{1-7} \mathrm{~A}$ pristine layer of graphene shows a gapless linear energy spectrum at two points of its Brillouin zone (namely $K$ and $K^{\prime}$ ) which prevents electron confinement and which complicates the fabrication of graphene-based electronic devices. ${ }^{6}$ This gapless behavior is a consequence of the sublattice (chiral) and time-reversal symmetries of graphene. It was recently found that the application of appropriate substrates can break the sublattice symmetry and induce a gap in the spectrum. ${ }^{8,9}$ The hexagonal boron-nitride (h-BN) substrate is such an example, and recent magnetotransport measurement of graphene on top of h-BN were very promising for high quality electronic devices. ${ }^{9}$ Furthermore, $a b$ initio density functional calculations (DFT) demonstrated that the energy spectrum of graphene exhibits a gap when placed on top of h-BN substrates. ${ }^{10,11}$ Such a breaking of the sublattice symmetry in graphene can be translated into a mass term in the Dirac-Weyl Hamiltonian. ${ }^{12}$ The spatial variation of such a mass term will be useful for the creation of quantum devices where a tunable energy gap allows the observation of confined states. ${ }^{13-15}$

Surface states have been investigated in topological insulator materials over the last three decades. Such materials are insulating in the bulk while on their boundaries topologically protected surface (edge) states are found. ${ }^{16}$ In this paper we propose one-dimensional (1D) chiral state in single layer graphene that relies on the creation of an asymmetric mass profile (i.e., a kink potential) [see Fig. 1(a)]. A similar electrostatic kink potential profile has been recently investigated in bilayer graphene (BLG), that can be realized using nanostructured double gates placed respectively on the bottom and top of the BLG layers. ${ }^{17-20}$ At the interface of the kink potential, 1D chiral states appear in the energy gap with unidirectional motion of the electrons. Recently, it was also found that these states are robust even in the presence of a perpendicular external magnetic field. ${ }^{21}$ In contrast to these early proposals our system can be realized with a single layer of graphene without using gate potentials. We show that these new chiral states can be induced by a line defect in the h-BN substrate that results in a kink in the effective-mass profile. Other types of line defects or vacancies can induce a mass profile different from the present kink-antikink structure which even may open an energy gap due to the coupling between the $K$ and $K^{\prime}$ valleys.

Here, we will solve the Dirac-Weyl Hamiltonian analytically, for the kink-antikink mass profile and obtain the dispersion relation explicitly. We complemented our results with DFT calculations. In our plane-wave base DFT calculations it is necessary to apply periodic boundary conditions. We use a rectangular graphene supercell containing 32 carbon atoms and place it on top of a boron-nitride substrate with a topological defect line [see Fig. 1(b)]. Different ways of stacking are possible but $A A$ stacking, i.e., all carbon atoms are on top of boron or nitrogen atoms, maximizes the graphene-substrate interaction. ${ }^{10}$ The DFT results support our analytical results and show that the line defect in h-BN results in the disappearance of the band gap which is normally induced by a homogeneous h-BN substrate. ${ }^{10}$

\section{KINK-SHAPED MASS PROFILE}

We employ the continuum model based on the Dirac equation, which in the valley isotropic form is described by ${ }^{7}$

$H=-i \hbar v_{F}\left[\begin{array}{cc}0 & \partial_{x}-i \partial_{y} \\ \partial_{x}+i \partial_{y} & 0\end{array}\right]+\tau\left[\begin{array}{cc}\Delta(x) & 0 \\ 0 & -\Delta(x)\end{array}\right]$,

where $v_{F}=10^{6} \mathrm{~m} / \mathrm{s}$ is the Fermi velocity, $\tau=1(\tau=-1)$ corresponds to the $K\left(K^{\prime}\right)$ valley, and $+\Delta(-\Delta)$ is the mass term induced by the BN substrate to the $A(B)$ sublattice. The mass term breaks the sublattice symmetry and a gap $2 \Delta$ opens in the graphene energy spectrum.

The eigenstates of Eq. (1) are two-component spinors $\psi=$ $\left[\phi_{a}(x, y), i \phi_{b}(x, y)\right]^{T}$ where $\phi_{a, b}$ are the envelope functions associated with the $A$ and $B$ sublattices. Since $\left[H,-i \partial_{y}\right]=0$, the momentum along the $y$ direction is a conserved quantity and we have $\psi=e^{i k_{y} y}\left[\varphi_{a}(x), i \varphi_{b}(x)\right]^{T}$, where $k_{y}$ is the wave vector along the $y$ direction. Solving the Schrödinger equation $H \psi(x, y)=E \psi(x, y)$ we obtain the two coupled differential equations as

$$
\begin{aligned}
{\left[\partial_{x}+k_{y}\right] \varphi_{b} } & =[\epsilon-\zeta(x)] \varphi_{a}, \\
-\left[\partial_{x}-k_{y}\right] \varphi_{a} & =[\epsilon+\zeta(x)] \varphi_{b} .
\end{aligned}
$$



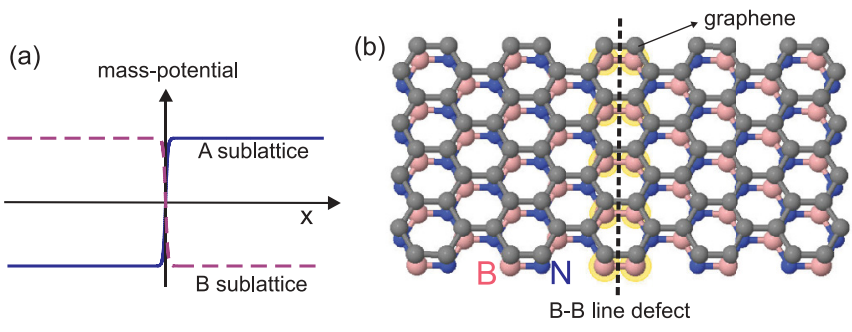

FIG. 1. (Color online) (a) The induced mass potential profile in single layer graphene in the presence of a boron-nitride $(\mathrm{BN})$ substrate with a line defect. (b) A schematic picture of graphene on top of $\mathrm{BN}$ substrate. The dashed line indicates the position of the line defect (i.e., B-B).

where $\epsilon=E l / \hbar v_{F}, \zeta(x)=\Delta(x) l / \hbar v_{F}$, and $(x, y) \rightarrow(x, y) / l$ are dimensionless with $l=1 \mathrm{~nm}$ taken as the length unit. For convenience we consider a steplike kink profile for the mass term which is modeled by $\zeta(x)=\zeta_{0} x /|x|$. We decouple Eqs. (2) and obtain

$$
\left[\partial_{x}^{2}-\gamma^{2}\right] \varphi_{a}=0
$$

where $\gamma=\sqrt{\zeta_{0}^{2}+k_{y}^{2}-\epsilon^{2}}$ is real when $|\epsilon|<\sqrt{\zeta_{0}^{2}+k_{y}^{2}}$ (i.e., the region where bound states exist). The solutions of Eq. (3) for $x>0$ and $x \leqslant 0$ regions are, respectively, given by

$$
\begin{aligned}
& \psi(x<0)=A e^{\gamma x}\left[1,\left(k_{y}-\gamma\right) /\left(\epsilon-\zeta_{0}\right)\right]^{T}, \\
& \psi(x>0)=B e^{-\gamma x}\left[1,\left(k_{y}+\gamma\right) /\left(\epsilon+\zeta_{0}\right)\right]^{T},
\end{aligned}
$$

where $\varphi_{b}$ is obtained using Eq. (2b). Matching the solution at $x=0$ leads to

$$
\epsilon \gamma=\zeta_{0} k_{y} .
$$

Equation (5) can be written as $\epsilon^{4}-\left(\zeta_{0}^{2}+k_{y}^{2}\right) \epsilon^{2}+\zeta_{0}^{2} k_{y}^{2}=0$ which results in four bands $\epsilon= \pm k_{y}$ and $\epsilon= \pm \zeta_{0}$. From Eq. (5) we notice that these solutions must satisfy $\operatorname{sgn}\left(k_{y} \epsilon\right)>0$ in addition to the $|\epsilon|<\sqrt{\zeta_{0}^{2}+k_{y}^{2}}$ condition. Notice that the flat bands at $\epsilon=-\zeta_{0}$ (for $k_{y}<0$ ) and $\epsilon=\zeta_{0}$ (for $k_{y}>0$ ) are not a solution for all $k_{y}$ values and should be discarded. From Eqs. (2) one can simply obtain the valid solution as $k_{y}=\zeta_{0}$ for $\epsilon=\zeta_{0}$ and $k_{y}=-\zeta_{0}$ for $\epsilon=-\zeta_{0}$. The equations for the $K^{\prime}$ valley can be obtained using the $\zeta(x) \rightarrow-\zeta(x)$ transformation in Eqs. (2) where it leads to the $\epsilon_{K^{\prime}}=-k_{y}$ solution for the $K^{\prime}$ valley.

Figure 2(a) shows the spectrum for a kinklike mass potential as function of the wave vector along the kink. The shaded region corresponds to the continuum of free states. The solid black curves correspond to the energy levels of a single layer graphene in the presence of a uniform mass potential $\zeta_{0}$ which can be obtained from Eq. (1) as $\epsilon= \pm \sqrt{k_{y}^{2}+\zeta_{0}^{2}}$. The gray dotted horizontal lines correspond to $\epsilon= \pm \zeta_{0}= \pm 0.152=(100 \mathrm{meV})$ and $\epsilon=0$. The solid blue line $\epsilon_{K}=k_{y}$ and the red dashed line $\left(\epsilon_{K^{\prime}}=-k_{y}\right)$ show the chiral states corresponding to the $K$ and $K^{\prime}$ valleys, respectively. Notice that these states have a unidirectional character of propagation, i.e., they are chiral states, with positive (negative) group velocity for the $K\left(K^{\prime}\right)$ valley. These states are related to the edge states of $2 \mathrm{D}$ electrons that are realized in the presence of a perpendicular magnetic field
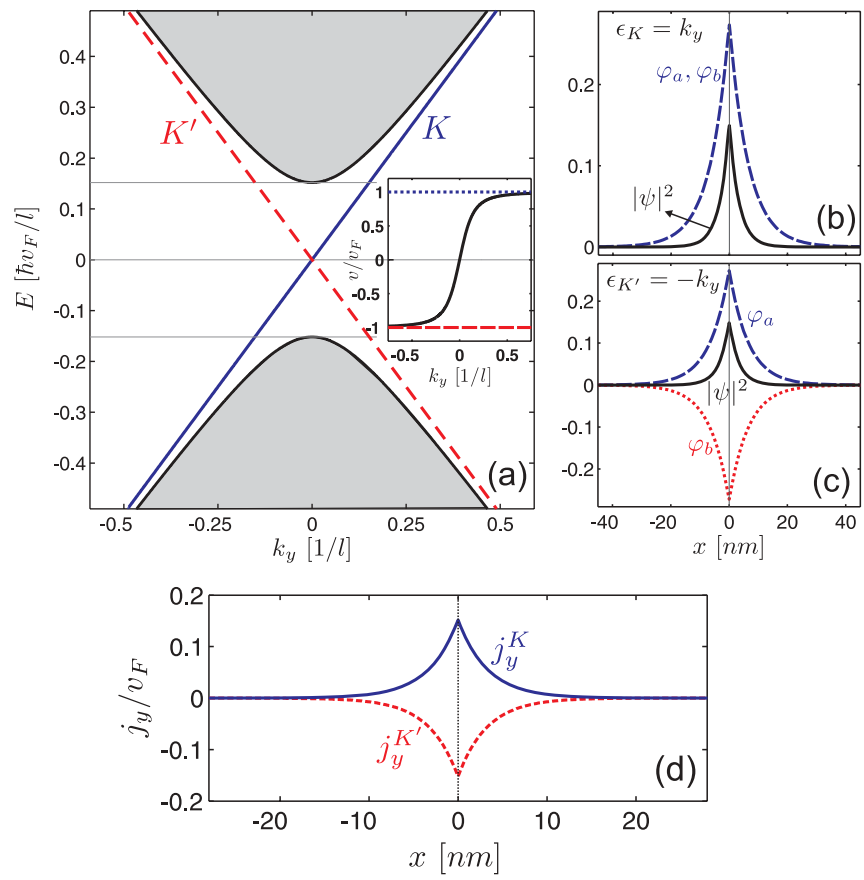

FIG. 2. (Color online) (a) Energy levels for a kink-shaped mass profile in single graphene. The curves and parameters are explained in the text. (b), (c) The wave spinors and probability density, respectively, for $\epsilon_{K}=k_{y}$ and $\epsilon_{K^{\prime}}=-k_{y}$. (d) $y$ component of the persistent current corresponding to the chiral state for the $K$ (blue solid curve) and the $K^{\prime}$ (red dashed curve) valleys as a function of the $x$ direction.

and that are responsible for the quantum Hall effect (QHE). Notice that here these states are realized without a magnetic field. The inset of Fig. 2(a) shows the velocity of the carriers for the chiral state which is $+v_{F}$ for the $K$ valley (blue dotted curve) and $-v_{F}$ for the $K^{\prime}$ valley (red dashed curve). The black solid curve displays the velocity of the electrons for a gapped single layer graphene, $\epsilon=\sqrt{k_{y}^{2}+\zeta_{0}^{2}}$, and results in the velocity $v / v_{F}=k_{y} / \sqrt{k_{y}^{2}+\zeta_{0}^{2}}$. Figures 2(b) and 2(c) present the real parts of the spinor components and the probability density for (a) $\epsilon_{K}=k_{y}$ and (b) $\epsilon_{K}=-k_{y}$. These electron states are localized at the position of the kink. Notice that the solutions corresponding to the $K$ and $K^{\prime}$ valleys are related by the transformations $\varphi_{a}^{K^{\prime}} \rightarrow-\varphi_{b}^{K}, \varphi_{b}^{K^{\prime}} \rightarrow \varphi_{a}^{K}$ and $k_{y}^{\prime} \rightarrow-k_{y}^{\prime}$. These unidirectional states move in the opposite direction when they belong to the $K$ and $K^{\prime}$ valley and therefore are very promising for electronic devices based on valleytronics. ${ }^{22,23}$ These chiral states exhibit persistent currents in the $y$ direction $j_{y}=\psi^{\dagger} \sigma_{y} \psi$ ( $\sigma_{y}$ is the $y$ component of the Pauli matrices). In Fig. 2(d), the $y$ component of the persistent current for the $K$ (blue solid curve) and $K^{\prime}$ (red dashed curve) valleys is shown as a function of the $x$ direction. Notice that the current is localized at the position of the kink interface and the $K$ and $K^{\prime}$ valleys carry the opposite currents.

\section{EFFECT OF SMALL GAP BETWEEN TWO MASS REGIONS}

In order to investigate the stability of these chiral states we consider the effect of a small gap between the two 

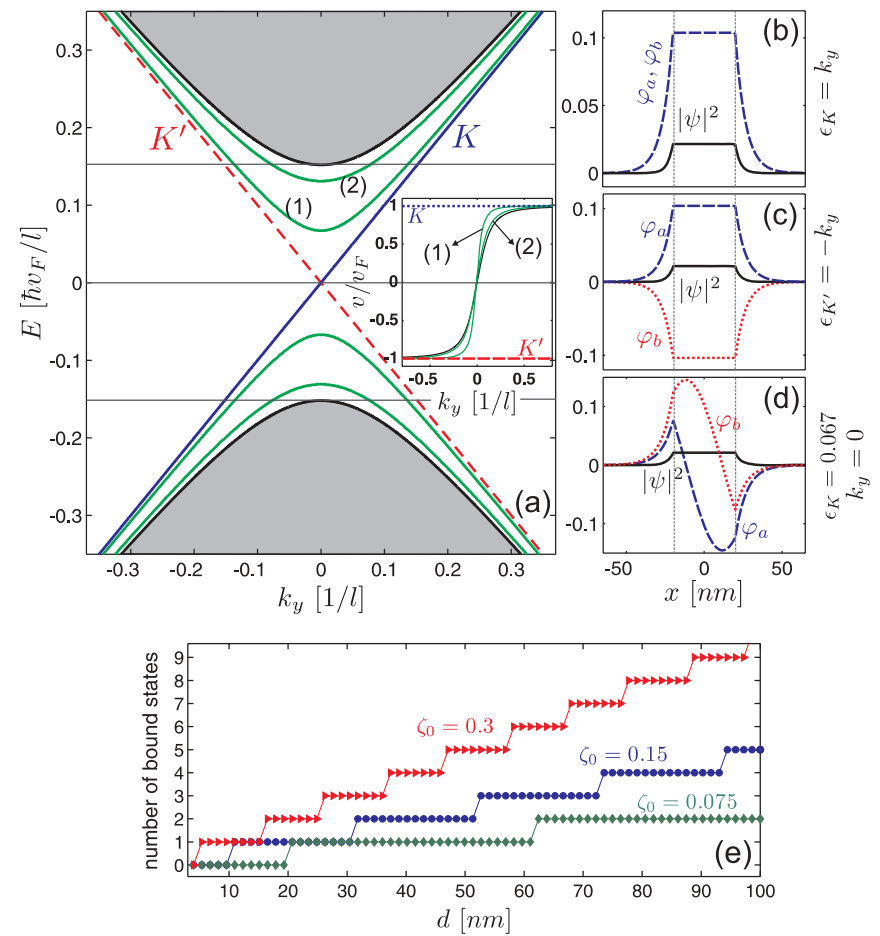

FIG. 3. (Color online) (a) Energy levels for a kink mass potential with a gap distance $d=40 \mathrm{~nm}$. (b)-(d) The wave spinors and corresponding probability density. (e) Number of additional bound states as a function of the gap distance. The curves and the parameters are explained in the text.

mass regions. We checked numerically that such a gap has qualitatively the same effect as smoothing the step profile of Fig. 1(a). We modeled the mass term by $\zeta(|x|>$ $d / 2)=\zeta_{0} x /|x|$ and $\zeta(|x| \leqslant d / 2)=0$. The eigenstates of Eq. (3) in the regions $I(x<-d / 2)$ and $I I I(x>d / 2)$ are given by Eqs. (4a) and (4b), respectively, and in the gap distance is $\psi_{I I}=C e^{i \lambda x}\left[1,\left(k_{y}-i \lambda\right) / \epsilon\right]^{T}+D e^{-i \lambda x}\left[1,\left(k_{y}+\right.\right.$ $i \lambda) / \epsilon]^{T}$, where $\lambda=\sqrt{\epsilon^{2}-k_{y}^{2}}$ and $d$ denotes the gap distance between the two mass regions. Matching the solutions at $x= \pm d / 2$ and setting the matrix of the coefficients to zero leads to the following equation:

$$
\left[\epsilon \gamma-\zeta_{0} k_{y}\right][\gamma \sin (d \lambda)+\lambda \cos (d \lambda)]=0 .
$$

The first part in Eq. (6) is the same as Eq. (5) (i.e., for the case of $d=0$ ) that leads to the above chiral states (i.e., $\epsilon_{K}=k_{y}$ and $\epsilon_{K^{\prime}}=-k_{y}$ ). Setting the second part between brackets to zero leads to a transcendental equation that results in extra localized states when $\epsilon>k_{y}$. These extra bound states for the $K^{\prime}$ valley can be obtained using the $\zeta_{0} \rightarrow-\zeta_{0}$ transformation in the second part of Eq. (6) which results in the same states as for the $K$ valley (i.e., $\epsilon_{K}=\epsilon_{K^{\prime}}$ for the extra localized states).

In Fig. 3(a) the energy levels as a function of $k_{y}$ are shown for the kink profile with a gap of $d=40 \mathrm{~nm}$ between the two mass regions. Notice that now in addition to the chiral states several branches (solid green curves) are seen which are split off from the continuum. Increasing the gap distance increases the number of those states that are confined near the kink. The number of these bound states can be related to the height of the mass potential $\zeta_{0}$. Figure 3(e) shows the number of these extra bound states for three different $\zeta_{0}$ values as a function of the gap distance $d$. The first bound state for $\zeta_{0}=0.075,0.15,0.3$ appears, respectively, at $d \approx 20,11,5 \mathrm{~nm}$. Notice that the chiral states $\epsilon_{K}=k_{y}$ (blue solid line) and $\epsilon_{K^{\prime}}=-k_{y}$ (red dashed line) are robust with regard to the smoothing of the step profile (here modeled by the gap distance). The velocity of the carriers corresponding to the chiral states (i.e., $v_{K}=v_{F}$ and $v_{K^{\prime}}=-v_{F}$ ) and the additional localized levels [labeled by (1) and (2)] are shown in the inset of Fig. 3. The extra bound states have a zero velocity at $k_{y}=0$ which is a consequence of the symmetric energy dispersion around $k_{y}=0$. The wave functions for $\epsilon_{K}=k_{y}$ and $\epsilon_{K^{\prime}}=-k_{y}$ are respectively shown in panels (b) and (c). In comparison with the sharp kink mass potential $(d=0)$ the probability density is now uniformly spread out over the distance $d$. Figure 3(d) shows the wave function for the first additional bound state [labeled by (1) in Fig. 3(a) ] at $k_{y}=0$ and $\epsilon_{K}=0.067$ which is also bound along the $x$ direction with a probability distribution very similar to the one of the chiral states but where the wave spinors exhibit now a nodal character in the $-d / 2 \leqslant x \leqslant d / 2$ region.

\section{SUPERLATTICE}

Now we extend our results to a superlattice (SL) of mass kinks. Here we use periodic boundary conditions $\psi(0)=$ $e^{i k_{x} L} \psi(L)$ where $L$ is the periodicity and $k_{x}$ denotes the wave vector along the $x$ direction. The kink mass interface is located at $x=L / 2$ which is repeated periodically. The solutions $\psi_{I}$ and $\psi_{I I}$, respectively for $x<L / 2$ and $x \geqslant L / 2$ regions, are given by

$$
\begin{aligned}
& \psi_{I}=A e^{\gamma x}\left[1, \frac{k_{y}-\gamma}{\epsilon-\zeta_{0}}\right]^{T}+B e^{-\gamma x}\left[1, \frac{k_{y}+\gamma}{\epsilon-\zeta_{0}}\right]^{T}, \\
& \psi_{I I}=C e^{\gamma x}\left[1, \frac{k_{y}-\gamma}{\epsilon+\zeta_{0}}\right]^{T}+D e^{-\gamma x}\left[1, \frac{k_{y}+\gamma}{\epsilon+\zeta_{0}}\right]^{T},
\end{aligned}
$$

where $\gamma=\sqrt{\zeta_{0}^{2}+k_{y}^{2}-\epsilon^{2}}$. Matching the solutions at $x=L / 2$ and imposing the periodic boundary condition we find a set of four algebraic equations that have a solution of

$$
\left[\left(\epsilon^{2}-k_{y}^{2}\right) \cosh (\gamma L)-\zeta_{0}^{2}\right]+\gamma^{2} \cos \left(k_{x} L\right)=0 .
$$

Figure 4(a) shows a 3D plot of the energy bands resulting from Eq. (8) as function of $k_{x}$ and $k_{y}$ near the $K$ point. Panels (b) and (c) are contour plots of $\epsilon_{K}\left(k_{x}, k_{y}\right)$ for the first and second conduction bands, respectively. Two solutions to Eq. (8) are $\epsilon_{K}= \pm \sqrt{k_{y}^{2}+\zeta_{0}^{2}}$ [see the upper and lower bands in Fig. 4(a)] which denotes the energy bands of gapped graphene with a uniform mass potential. Another solution results in a Dirac cone at $\left(k_{x}, k_{y}\right)=\mathbf{0}$ which is a consequence of the additional states brought by the antikink steps in the SL system [see intermediate bands in Fig. 4(a)]. Expanding Eq. (8) for $k_{x}, k_{y} \rightarrow 0$ and $\epsilon \ll \sqrt{k_{y}^{2}+\zeta_{0}^{2}}$ we obtain $\epsilon_{k}= \pm \sqrt{k_{x}^{2}+k_{y}^{2}}$ which demonstrates the linearity of the dispersion relation around the Dirac cone. Such Dirac cones have also been found in graphene superlattices. ${ }^{24}$

\section{AB INITIO SIMULATIONS}

We supplemented our theoretical treatment with $a b$ initio DFT simulations. We used the ABINIT software package ${ }^{25}$ to 

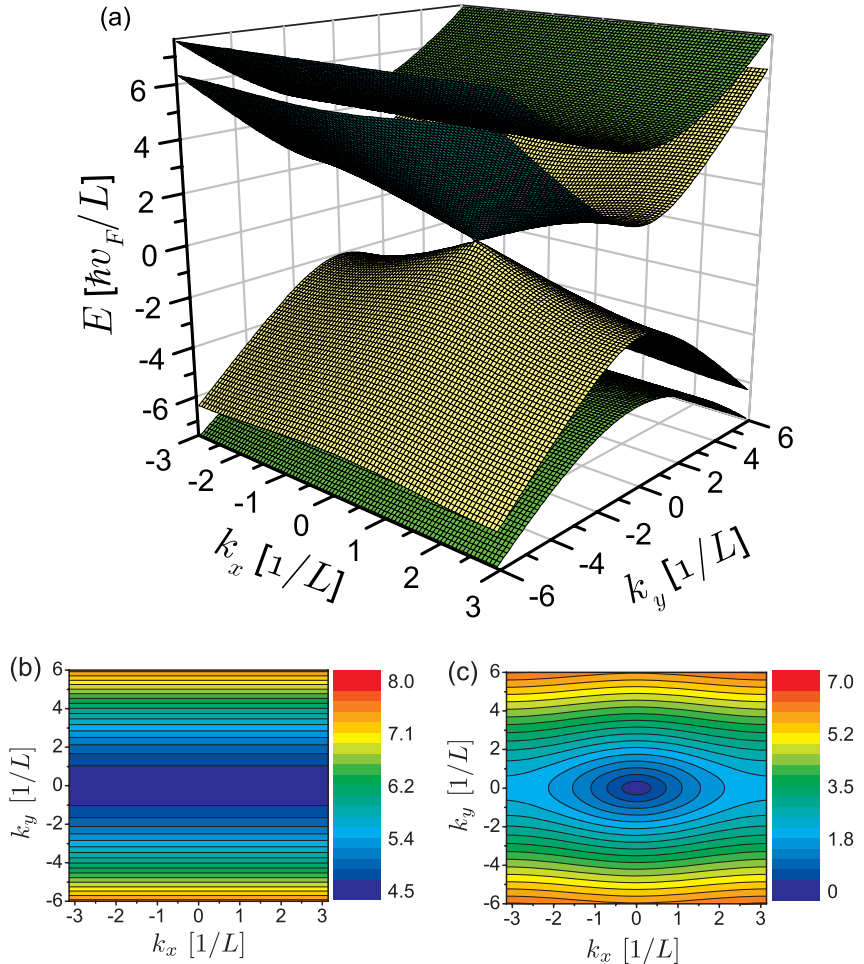

FIG. 4. (Color online) (a) 3D plot of the energy bands in the presence of a superlattice of kink mass potentials in single layer graphene with $\zeta_{0}=4.55(=100 \mathrm{meV})$ and $L=30 \mathrm{~nm}$. Panels (b) and (c), respectively, display the contour plots corresponding to the first and the second conduction bands.

calculate the electronic band structure of graphene on top of a boron-nitride substrate with and without the line defect. These calculations were done within the local-density approximation (LDA) to obtain a reasonable description of the graphene-BN interlayer interaction ${ }^{26}$ and the specific parameters for the DFT calculations were the same as given in Ref. 26. The specific model structure that we used consists of a rectangular graphene supercell containing $32 \mathrm{C}$ atoms on top of a $\mathrm{BN}$ substrate where the relaxed distance between the $\mathrm{BN}$ substrate and graphene is set to $3.24 \AA$ [see Fig. 5(c)]. The line of defects are taken along the zigzag direction and consists of alternating B-B and N-N defect lines separated by defect-free $\mathrm{h}-\mathrm{BN}$ regions with a width of approximately $2 \mathrm{~nm}$. In Figs. 5(a) and 5(b), the electronic band structure of the investigated systems, calculated along the $k_{y}$ direction and through the $\Gamma$ point, is shown. As can be seen from Fig. 5(a), a band gap, i.e., $\approx 56 \mathrm{meV}$, is opened when graphene is placed on top of a perfect BN substrate. When the line of defects, corresponding to a kink in the mass potential, is added, the band gap closes and the linear spectrum at the $K$ point is recovered [see Fig. 5(b)]. This is in agreement with the analytical results derived before. Note that there are some differences between the band spectra as obtained with DFT and the analytical result for a superlattice [Figs. 5(b) and 4, respectively]: (i) There are additional bands in the DFT spectrum because of the explicit incorporation of the boronnitride substrate in the calculations. (ii) The parabolic bands are shifted to a larger $k_{y}$ point (i.e., away from the $K$ point) and are situated farther away from the Fermi level. This can
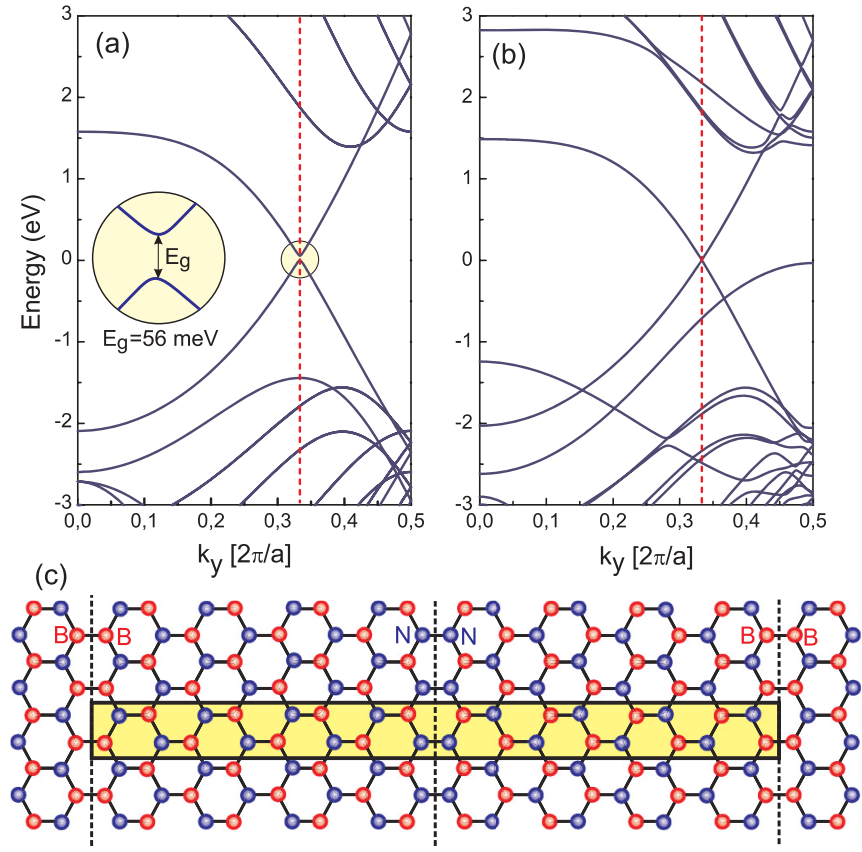

FIG. 5. (Color online) The band structure obtained within DFT for (a) graphene on top of a uniform layer of a $\mathrm{BN}$ substrate and (b) graphene on top of a BN substrate with a periodic area of line defects. The vertical dashed line indicates the position of the $K$ point. The structure that is simulated is shown in (c): the supercell is indicated by the yellow rectangle and the dashed vertical lines indicate the position of the line defects.

be attributed to the relatively small size of the supercell in the DFT calculations as compared to the continuum model.

\section{CONCLUDING REMARKS}

In summary, we predicted new chiral states in the presence of an asymmetric (kink profile) mass potential in single layer graphene. Our study showed that these states are robust even when a small gap is located between the two mass regions. The system can be realized experimentally using a boron-nitride substrate with a line defect that induces an asymmetric mass potential in graphene. These 1D chiral states were also investigated within $a b$ initio calculations for a superlattice of line defects. Throughout our calculations we showed that the chiral states corresponding to the $K$ and $K^{\prime}$ valleys exhibit the opposite direction of propagation which is a consequence of their chiral nature. Nanostructuring of such line defects in the h-BN substrate can be very promising for the fabrication of electronic devices for valleytronics. Graphene dots with perfect zigzag edges were earlier proposed for valleytronics applications. ${ }^{22}$ The realization of perfect zigzag edges is very challenging and this disadvantage does not arise in our case, where the asymmetric mass potential is induced by a line of defects in the h-BN substrate.

\section{ACKNOWLEDGMENTS}

This work was supported by the Flemish Science Foundation (FWO-Vl), and the European Science Foundation (ESF) under the EUROCORES Program: EuroGRAPHENE (project CONGRAN). 
${ }^{1}$ K. S. Novoselov, A. K. Geim, S. V. Morozov, D. Jiang, Y. Zhang, S. V. Dubonos, I. V. Grigorieva, and A. A. Firsov, Science 306, 666 (2004).

${ }^{2}$ K. S. Novoselov, A. K. Geim, S. V. Morozov, D. Jiang, M. I. Katsnelson, I. V. Grigorieva, S. V. Dubonos, and A. A. Firsov, Nature (London) 438, 197 (2005).

${ }^{3}$ Y. Zheng and T. Ando, Phys. Rev. B 65, 245420 (2002).

${ }^{4}$ V. P. Gusynin and S. G. Sharapov, Phys. Rev. Lett. 95, 146801 (2005).

${ }^{5}$ Y. Zhang, Y. W. Tan, H. L. Stormer, and P. Kim, Nature (London) 438, 201 (2005).

${ }^{6}$ A. H. Castro Neto, F. Guinea, N. M. R. Peres, K. S. Novoselov, and A. Geim, Rev. Mod. Phys. 81, 109 (2009).

${ }^{7}$ C. W. J. Beenakker, Rev. Mod. Phys. 80, 1337 (2008).

${ }^{8}$ S. Y. Zhou, G.-H. Gweon, A. V. Fedorov, P. N. First, W. A. de Heer, D.-H. Lee, F. Guinea, A. H. Castro Neto, and A. Lanzara, Nat. Mater. 6, 770 (2007).

${ }^{9}$ C. R. Dean, A. F. Young, I. Meric, C. Lee, L. Wang, S. Sorgenfrei, K. Watanabe, T. Taniguchi, P. Kim, K. L. Shepard, and J. Hone, Nat. Nanotechnol. 5, 722 (2010).

${ }^{10}$ G. Giovannetti, P. A. Khomyakov, G. Brocks, P. J. Kelly, and J. van den Brink, Phys. Rev. B 76, 073103 (2007).

${ }^{11}$ B. Sachs, T. O. Wehling, M. I. Katsnelson, and A. I. Lichtenstein, Phys. Rev. B 84, 195414 (2011).

${ }^{12}$ P. Recher, J. Nilsson, G. Burkard, and B. Trauzettel, Phys. Rev. B 79, 085407 (2009).

${ }^{13}$ S. Schnez, K. Ensslin, M. Sigrist, and T. Ihn, Phys. Rev. B 78, 195427 (2008).
${ }^{14}$ M. Zarenia, J. M. Pereira, A. Chaves, F. M. Peeters, and G. A. Farias, Phys. Rev. B 81, 045431 (2010).

${ }^{15}$ M. Grujic, M. Zarenia, A. Chaves, M. Tadic, G. A. Farias, and F. M. Peeters, Phys. Rev. B 84, 205441 (2011).

${ }^{16}$ B. A. Volkov and Q. A. Pankratov, Pis'ma Zh. Eksp. Teor. Fiz. 42, 145 (1985) [JETP Lett. 42, 178 (1985)].

${ }^{17}$ I. Martin, Ya. M. Blanter, and A. F. Morpurgo, Phys. Rev. Lett. 100, 036804 (2008)

${ }^{18}$ M. Killi, T.-C. Wei, I. Affleck, and A. Paramekanti, Phys. Rev. Lett. 104, 216406 (2010).

${ }^{19}$ L. Jing, J. Velasco Jr., P. Kratz, G. Liu, W. Bao, M. Bockrath, and C. N. Lau, Nano Lett. 10, 4775 (2010).

${ }^{20}$ Zh. Qiao, J. Jung, Q. Niu, and A. H. MacDonald, Nano Lett. 11, 3453 (2011).

${ }^{21}$ M. Zarenia, J. M. Pereira Jr., G. A. Farias, and F. M. Peeters, Phys. Rev. B 84, 125451 (2011).

${ }^{22}$ A. Rycerz, J. Tworzydlo, and C. W. J. Beenakker, Nat. Phys. 3, 172 (2007).

${ }^{23}$ J. Jung, F. Zhang, Zh. Qiao, and A. H. MacDonald, Phys. Rev. B 84, 075418 (2011)

${ }^{24}$ M. Barbier, P. Vasilopoulos, and F. M. Peeters, Phys. Rev. B 81, 075438 (2010)

${ }^{25}$ X. Gonze, J.-M. Beuken, R. Caracas, F. Detraux, M. Fuchs, G.-M. Rignanese, L. Sindic, M. Verstraete, G. Zerah, F. Jollet, M. Torrent, A. Roy, M. Mikami, P. Ghosez, J.-Y. Raty, and D. C. Allan, Comput. Mater. Sci. 25, 478 (2002).

${ }^{26}$ O. Leenaerts, B. Partoens, and F. M. Peeters, Phys. Rev. B 80, 245422 (2009) 